\title{
The performance of the Expanded Programme on Immunization in a rural area of Mozambique
}

Miguel Lanaspa * a,b ${ }^{\text {a }}$, Reyes Balcells ${ }^{\mathrm{a}, \mathrm{b}}$, Charfudin Sacoor ${ }^{\mathrm{b}}$, Abel Nhama ${ }^{\mathrm{b}}$, John J Aponte ${ }^{\mathrm{a}}$, and Quique Bassat ${ }^{\mathrm{a}, \mathrm{b}}$

* Corresponding author

a Barcelona Centre for International Health Research (CRESIB, Hospital ClínicUniversitat de Barcelona), Barcelona, Spain

Roselló 132, 4

08036 Barcelona, Spain

Tel: + 932275706

b Centro de Investigação em Saúde de Manhiça (CISM), Maputo, Mozambique

Bairro Cambeve, Rua 12, Distrito da Manhiça

CP 1929, Maputo, Mozambique

Tel: +25821810002

Email addresses (in order of authorship)

miguel.lanaspa@cresib.cat (corresponding author)

reyesbalcells@gmail.com

charfudin.sacoor@manhica.net

abel.nhama@manhica.net

john.aponte@cresib.cat

quique.bassat@cresib.cat 


\section{ABSTRACT}

Vaccines are an effective public health measure. Vaccination coverage has improved in Africa in the last decades but has still not reached WHO/UNICEF target of at least $90 \%$ first-dose coverage for vaccines in the Expanded Programme on Immunization (EPI) implemented in Mozambique in 1979. There are concerns about reliability of vaccination coverage official data from low-income countries, and inequities in vaccine administration. We randomly sampled 266 under-five years children from Taninga, a poor rural area in Southern Mozambique under a Demographic Surveillance System and collected data directly from the individual national health cards when available (BCG, DTP/HepB/Hib, Polio, Measles). We also collected data on socio-economic variables through an interview. Overall, only $5 \%$ of the participants did not receive all the doses of the vaccines included in the EPI in a timely manner (overall vaccination coverage 95\%, 95\%CI: 93.5\%-95.5\%). The socio-economic status was homogenously low and no differences were found between vaccinated and unvaccinated children. Vaccination coverage in Taninga was very high, despite the low socioeconomic status of the population. The high performance of the EPI in Taninga is an encouraging experience for achieving high vaccination coverage in low-income rural settings.

\section{KEYWORDS}

Immunization Programs; Vaccination coverage; DTP3; Children; Developing Countries. 
59

60

61

62

63

64

65

66

67

68

69

70

71

72

73

74

75

76

77

78

79

80

81

\section{BACKGROUND}

Vaccines are amongst the most cost-effective public health measures currently available in developing countries (Deogaonkar et al., 2012) where they prevent 2.5 annual million deaths (WHO et al., 2009). Vaccination coverage has improved during the last decade. As an example, average coverage of DTP3 (3 doses of diphtheria, tetanus and pertussis vaccine) was 50\% in Africa in 1996 and 72\% in 2012 (World Health Organization and UNICEF, 2013). A reason for this is that, since 2000, the Global Alliance for Vaccines and Immunisation (GAVI) has supported a growing number of countries (73 in 2010) through a performance-based grant programme to achieve WHO/UNICEF recommended target: at least $90 \%$ first-dose coverage for the vaccines included in the Expanded Programme of Immunization (EPI) in 2015 (Le Gargasson et al., 2013; United Nations, 2013). The "Reaching Every District" (RED) WHO approach, implemented in Africa since 2002, has also contributed to improve childhood immunization services increasing coverage rates from $73 \%$ to $94 \%$ for different antigens in 2009 worldwide (Tao et al., 2013). The continuous uptake of vaccination will avert over 20 million deaths during the 2011-2020 period worldwide, 52\% of which will be in African countries (Lee et al., 2013).

Despite the encouraging figures, two main concerns have arisen. First, different authors have questioned the quality and validity of the official data from low-income countries (BoschCapblanch et al., 2009; Murray et al., 2003; Ronveaux et al., 2005). The verification factor quantifies under or over-reporting of vaccine doses at a district level. It is based on the ratio of re-counted DTP3 doses in the health unit records by the number of DTP3 doses reported by the health unit to the district (Ronveaux et al., 2005). Audits of official data in countries from Africa, America, and Asia show a low verification factor in vaccine administration, which hampers the ability to track the performance of the programmes and to detect failures and 
weaknesses (Bosch-Capblanch et al., 2009; Murray et al., 2003; Ronveaux et al., 2005). Furthermore, the official data of vaccination coverage is 20 percentage points superior to data obtained through Health and Demographic Surveillance Systems (Murray et al., 2003; Tao et al., 2013). The second concern is that global figures could hide inequities in public health interventions coverage, which could jeopardize the achievement of Millennium Development Goals (MDG) 1 -Eradicate extreme poverty and hunger- and 4 -Reduce child mortality(Lauridsen and Pradhan, 2011; United Nations, 2013). Regarding immunization (DTP3, Measles, fully immunized), differences of coverage between the poorest and the richest quintile from the same country can be as considerable as 20\% (Barros et al., 2012; United Nations, 2013). Determinants impairing vaccination coverage are distance to vaccination site, waiting time, unavailability of treatment services, cost, low parental education level and large family size (Falagas and Zarkadoulia, 2008; Nkonki et al., 2011; Rainey et al., 2011; Van Malderen et al., 2013).

Mozambique is one of the poorest countries in the world. In 2011, it had the fourth lowest Human Development Index worldwide (UNPD, 2013). The EPI was introduced in Mozambique in 1979, and the RED approach in 2008. However, RED has only been implemented in 66 out of 144 Mozambican districts (Ministry of Health, 2011). The verification factor of the vaccines administered (DTP3) was less than 0.70 in 2002-2003, which implies over-reporting and inability to verify the administration of DTP3 (Ronveaux et al., 2005). Furthermore, among 54 countries monitored by Countdown to 2015, Mozambique was the $14^{\text {th }}$ in terms of income-specific inequity in measles vaccine administration (Barros et al., 2012). The official national estimate for DTP3 coverage was 76\% in 2011 (GAVI Alliance, 2013). Administrative data showed DTP3 coverage of 49.2\% in Maputo province, and $90.2 \%$ in Maputo city, which provides an idea of differences in rural and urban settings 
110 (Ministry of Health, 2011). It is important to determine the validity of this figure before

111 introducing new vaccines.

112

113 The aim of the study was to determine the coverage of the EPI vaccines in a rural area of

114 southern Mozambique included in a Demographic Surveillance System (DSS), and to

115 describe the socio-economic determinants of vaccination within this area.

116 


\section{MATERIAL AND METHODS}

\section{$118 \quad$ 2.1. Study population}

119 The study was conducted by the Centro de Investigação em Saúde de Manhiça (CISM),

120 located in Manhiça, Maputo Province, Southern Mozambique from September to November

121 2012. CISM runs a DSS in its study area and a morbidity surveillance system at Manhiça

122 District Hospital (MDH) and other health posts in the area. Manhiça District Hospital and the

123 peripheral health posts are facilities from the national public health system that receive 124 support from CISM. The DSS covers a 500- $\mathrm{km}^{2}$ area with 90,000 people, who are

125 individually given a unique and permanent identification number, and 18,000 households,

126 which are geopositioned. A field worker fills a demographic questionnaire at each household

127 at least once a year. Independently from the DSS, each child has a national health card with

128 information about vaccination. Taninga is a rural area of the DSS $30 \mathrm{~km}$ north from Manhiça,

129 and it only counts with one health post providing clinical assistance to the neighbouring

130 population. CISM does not directly intervene in EPI administration, but it provides support to

131 Taninga health post with health workers (medical agents, microscopists and nurses) since

132 2005. Since then, the health post has been open and available 24/7 for medical assistance.

133 CISM has also established community councils with community leaders of the study area on a

134 regular basis. Taninga was chosen as the site for this study as example of a very rural area. At

135 the time of the study, there were 1096 children under five years of age in Taninga according 136 to the DSS.

\section{$138 \quad$ 2.2. Selection of participants}

139 This sub-analysis is part of a larger study designed to determine the prevalence of viral

140 infections among healthy children in the community, approved by the Institutional Ethics

141 Committee of CISM, the National Ethics Committee of Mozambique, and the Institutional 
142 Review Board of Hospital Clinic in Barcelona. Sample size was calculated according to the

143 primary endpoint of the larger study, and thus was not based on any predefined hypothesis on

144 expected vaccination coverage rates. We obtained the sampling frame selecting from the DSS

145 database all the children aged less than 5 years living in Taninga. Selection of participants

146 was done by simple random sampling using STATA with the permanent identification

147 number as identifier. A field worker visited the participant household to invite the legal

148 guardian and the child. If the legal guardian was absent during the visit, a maximum of two

149 more visits were done to invite the child. In case of repeated absence or refusal to participate

150 in the study, another child of similar age and household location was approached. If the

151 guardians accepted the invitation, they were taken with their child to the Taninga health post

152 on the next day. Guardians were interviewed and children examined only after they had

153 signed the informed consent form.

\subsection{Vaccines received and definitions of correct vaccination}

156 The EPI in Mozambique includes a Bacille Calmette-Guerin (BCG) and an Oral Polio

157 Vaccine (OPV) dose at birth, a tetra/pentavalent (Hepatitis B, Diphteria, Tetanus, Pertussis, 158 plus Haemophilus influenzae $b$ since august 2009) at weeks 6, 10 and 14, and a Measles 159 vaccine single dose at month 9 (Table 1). Pneumococcal conjugate vaccine (PCV-10) has

160 recently been introduced $\left(10^{\text {th }}\right.$ April 2013) but was unavailable at the time of the study.

161 Vaccination is scheduled with the mother at child's birth when the first doses are given and

162 successive vaccinations are done at the nearest health post and recorded in the patient's

163 individual national health card. With the exception of Maputo, the country's capital, there is

164 virtually no private sector in which vaccines can be obtained outside the public health sector.

165 Only valid doses were considered when estimating vaccination coverage, that is, only age-

166 appropriate doses administered that respected recommended intervals between doses. A delay 
167 in vaccination less than 15 days was considered age-appropriate. Data on vaccination was

168 obtained from vaccination cards examined by the study clinician.

\subsection{Other data}

171 Years of education and wealth were used as proxies of socio-economic status. Data on

172 household assets and amenities were collected as proxies of wealth. A principal component

173 analysis was performed to determine which assets could be used to build a meaningful SES

174 index. Socio-economic data were obtained through an interview of the legal guardian

175 conducted by a field worker fluent in the local language (changana). Variables selected were

176 (i) related to the house; (ii) related to facilities (water source, availability of electricity, fuel

177 used to cook); and (iii) related to parental education and employment. The education level

178 (primary, secondary, superior) was defined as having completed at least 1 year of primary,

179 secondary, or superior education. Employment was defined as receiving a salary for their

180 professional activity, thus excluding subsistence farming.

181 Children were examined by the study clinician, and measured and weighted by the study 182 nurse. Mothers' HIV status was recorded from the vaccination cards. Distance to health 183 facility was calculated using the households and Taninga health post coordinates with 184 ArcGIS® version 9.3.

\subsection{Data management and statistical analysis}

187 Questionnaires were double entered in the study database using OpenClinica. Nutritional

188 status was based on weight-for-age Z scores (WAZ), calculated using the least mean square method and the 2000 CDC Growth Reference (Centers for Disease Control and Prevention, 2000). Data were analysed using Stata ${ }^{\circledR}$ (Stata Corp., College Station, TX, USA; version 12.0). The chi-square test and the t-student test were used when appropriate. 
193

194

195

196

197

\section{RESULTS}

\subsection{Population and socio-economic data}

We approached 266 children and recruited 206 (Figure 1). Among participants, mild malnutrition was frequent (31.7\%), $20.4 \%$ of the mothers were HIV positive and $86.6 \%$ were unemployed (Table 2). Overall, $38.5 \%$ of the mothers were uneducated, and only $11 \%$ had fulfilled at least one year of secondary school. Regarding facilities, $96.5 \%$ of the households had traditional latrines, $98.5 \%$ had the cooking space located outside, and $97.9 \%$ used wood for cooking.

\subsection{Vaccination coverage}

Two hundred (97.1\%) vaccination cards were available. Among them, 190 (95\%) children had correct vaccination for their age considering number and timing of doses (Table 3).

There were no significant differences on vaccination coverage by sex (coverage among males 94.3\%; coverage among females $95.7 \%$; $=0.64)$. Vaccination coverage was consistently high by age group (95\% in the younger group, $100 \%$ in the $6-12$ months group, and $93.5 \%$ in the oldest groups). The DTP3 coverage (coverage of third dose of DTP, variable used by GAVI and WHO/UNICEF) was calculated for comparison purposes. Among 178 children of complete DTP vaccination age (14 weeks of age plus 15 days of accepted vaccination delay), the DTP3 coverage was $98.3 \%$ (95\% CI: $97.35 \%-99.3 \%)$.

\subsection{Association between vaccination coverage and socio-economic data}

The distribution of socio-economic variables within different households was exceedingly uniform, hindering the development of a reliable socio-economic score through principal component analysis (Table 4). Combined with this socio-economic uniformity, the number of non-vaccinated children was too low to allow proper comparisons of socio-economic 
218 variables between groups. However, children from households with piped water had 219 significantly higher vaccination coverage than children from households obtaining water from 220 a well (97.6\% (120/123) vs. 90.9\% (70/77); $\mathrm{p}=0.036)$. 


\section{DISCUSSION}

224

According to official data from Mozambique's government (Demographic National Questionnaire 2011), vaccination coverage in rural areas nationwide was $89.9 \%$ for BCG, 72.4\% for DTP3, $70.9 \%$ for OPV, and $77.7 \%$ for measles. Compared to national vaccination coverage in rural areas, the vaccination coverage found in this study is very high. Inequity in vaccination affects illiterate, poor families especially if they have 3 or more children (Lauridsen and Pradhan, 2011; Nkonki et al., 2011; Van Malderen et al., 2013). Taninga is a rural area where the main economic activity is subsistence farming. Men from Taninga frequently migrate to South Africa to work in the mining industry that employed up to 70,000 Mozambican miners from Maputo and Gaza provinces in 2012 (Barwise et al., 2013). Study children belong to low-income, uneducated, large families, which make them vulnerable regarding to vaccination coverage. Our data show a different and unexpected situation. The vaccination coverage of the oldest group reflects good immunisation services even 5 years before the survey was done.

In poor populations, indirect costs of vaccination such as day of work loss and transport costs are dissuasive for the families, even if vaccination itself is free (Falagas and Zarkadoulia, 2008; Rainey et al., 2011). A clustered randomised trial in a rural area in India showed a vaccination coverage of $39 \%$ in the villages where a humble incentive was given to the families ( $1 \mathrm{~kg}$ of raw lentils and a set of metal plates) in the immunisation camps when their children were vaccinated compared to a coverage of $6 \%$ in the villages where no incentive was given nor immunisation camps were held (Banerjee et al., 2010). In Taninga, no incentive is offered to the families and the indirect costs of vaccination do not seem to dissuade families to have their children vaccinated. In the study done in India, the reliability of the 
247 immunisation camps held monthly seemed to be an encouraging factor for vaccination. In the

248 villages where reliable immunisation camps were held but no incentive was given, the

249 vaccination coverage was significantly higher than the coverage in villages without

250 intervention (Banerjee et al., 2010). In an econometric study based on data from DSS from 49

251 developing countries including Mozambique, the authors conclude that a higher density of

252 health workers, especially nurses, is related to higher vaccination coverage (Anand and 253 Barnighausen, 2007). The effect of reliability of health facilities on vaccination coverage 254 found in the Indian trial might also apply in a Sub-Saharan setting, although we have no data 255 on vaccination coverage before 2005 to support this hypothesis. The effect of health 256 information provided to the communities on vaccines acceptance has not been assessed in 257 Taninga.

259 Data of this study are encouraging for the success of pneumococcal vaccine introduction in 260 Taninga, which was implemented in April 2013, although national vaccination coverage 261 cannot be extrapolated from our study. High coverage benefits directly immunised children 262 but also the community through herd immunity. As a recent experience, the Haemophilus 263 influenza b (Hib) vaccine introduction in August 2009 led to a significant fall of the number 264 of children admitted to the Manhiça District Hospital with 24 Hib positive blood cultures per 265 year prior the introduction of the vaccine (2001-2009) down to 4.9 cases per year after the 266 introduction (2010-2012, unpublished data).

268 This study has some limitations. Firstly, comparison between groups is difficult due to the sample size, uniformity of socio-economic determinants and high vaccination coverage. In any case, vaccination coverage and socio-economic data are valid for descriptive purposes

271 since the sample was obtained through simple random sampling of a DSS determined 
272 population. The second limitation is the limited external validity of these data as the 273 population in Taninga is not representative of the whole population in Mozambique, but this 274 was not the objective of the study. Vaccination coverage could have been overestimated due

275 to the exclusion of 6 children without vaccination cards. If all of them were incorrectly 276 vaccinated, the vaccination coverage would be $92.2 \%(190 / 206)$, which is still higher than 277 expected. Finally, this study cannot determine reasons (such as being part of a DSS area and 278 the special attention it provides) for this unexpected high vaccination coverage.

\section{CONCLUSIONS}

281 Vaccination coverage in Taninga is very high, despite its population being particularly 282 vulnerable to low coverage in terms of socio-economic determinants (poor, uneducated, large 283 families in a rural sub-Saharan area). Understanding the particularities of this population 284 could be useful to improve the vaccination coverage nationwide, and should be explored in an 285 appropriate study. The high performance of the EPI in Taninga is an encouraging experience 286 for achieving high vaccination coverage in low-income rural settings. 


\section{6. ACKNOWLEDEGEMENTS}

288 Funding: This study was conducted as part of a larger study funded by the Bill and Melinda 289 Gates Foundation (OPP500092) to discover biomarkers to differentiate malaria from bacterial 290 and viral pneumonia.

291 We thank Humberto Mucasse, Albertina Manhiça, Alice Chitlango, Valente Melembe and 292 Enfermeiro Elidio for their dedication in the study. We are also thankful to the community of 293 Taninga for their insights during the meeting before the study. We especially thank children 294 and parents for their participation. 


\section{REFERENCES}

Anand, S., Barnighausen, T., 2007. Health workers and vaccination coverage in developing countries: an econometric analysis. Lancet 369, 1277-1285.

Banerjee, A.V., Duflo, E., Glennerster, R., Kothari, D., 2010. Improving immunisation coverage in rural India: clustered randomised controlled evaluation of immunisation campaigns with and without incentives. Bmj 340, c2220.

Barros, A.J., Ronsmans, C., Axelson, H., Loaiza, E., Bertoldi, A.D., Franca, G.V., Bryce, J., Boerma, J.T., Victora, C.G., 2012. Equity in maternal, newborn, and child health interventions in Countdown to 2015: a retrospective review of survey data from 54 countries. Lancet 379, 1225-1233.

Barwise, K., Lind, A., Bennett, R., Martins, E., 2013. Intensifying action to address HIV and tuberculosis in Mozambique's cross-border mining sector. International journal of health services : planning, administration, evaluation 43, 699-719.

Bosch-Capblanch, X., Ronveaux, O., Doyle, V., Remedios, V., Bchir, A., 2009. Accuracy and quality of immunization information systems in forty-one low income countries. Tropical medicine \& international health : TM \& IH 14, 2-10.

Centers for Disease Control and Prevention, 2000. CDC growth charts.

Deogaonkar, R., Hutubessy, R., van der Putten, I., Evers, S., Jit, M., 2012. Systematic review of studies evaluating the broader economic impact of vaccination in low and middle income countries. BMC public health 12, 878 .

Falagas, M.E., Zarkadoulia, E., 2008. Factors associated with suboptimal compliance to vaccinations in children in developed countries: a systematic review. Current medical research and opinion $24,1719-1741$.

GAVI Alliance, 2013. GAVI Alliance Progress Report 2013. 
Lauridsen, J., Pradhan, J., 2011. Socio-economic inequality of immunization coverage in India. Health economics review 1, 11.

Le Gargasson, J.B., Breugelmans, J.G., Mibulumukini, B., Da Silva, A., Colombini, A., 2013. Sustainability of National Immunization Programme (NIP) performance and financing following Global Alliance for Vaccines and Immunization (GAVI) support to the Democratic Republic of the Congo (DRC). Vaccine 31, 1886-1891.

Lee, L.A., Franzel, L., Atwell, J., Datta, S.D., Friberg, I.K., Goldie, S.J., Reef, S.E., Schwalbe, N., Simons, E., Strebel, P.M., Sweet, S., Suraratdecha, C., Tam, Y., Vynnycky, E., Walker, N., Walker, D.G., Hansen, P.M., 2013. The estimated mortality impact of vaccinations forecast to be administered during 2011-2020 in 73 countries supported by the GAVI Alliance. Vaccine 31 Suppl 2, B61-72.

Ministry of Health, 2011. National Immunization Programme. Comprhensive Multi-Year Plan 2012 - 2016, Mozambique.

Murray, C.J., Shengelia, B., Gupta, N., Moussavi, S., Tandon, A., Thieren, M., 2003. Validity of reported vaccination coverage in 45 countries. Lancet 362, 1022-1027.

Nkonki, L.L., Chopra, M., Doherty, T.M., Jackson, D., Robberstad, B., 2011. Explaining household socio-economic related child health inequalities using multiple methods in three diverse settings in South Africa. International journal for equity in health 10, 13.

Rainey, J.J., Watkins, M., Ryman, T.K., Sandhu, P., Bo, A., Banerjee, K., 2011. Reasons related to non-vaccination and under-vaccination of children in low and middle income countries: findings from a systematic review of the published literature, 1999-2009. Vaccine $29,8215-8221$.

Ronveaux, O., Rickert, D., Hadler, S., Groom, H., Lloyd, J., Bchir, A., Birmingham, M., 2005. The immunization data quality audit: verifying the quality and consistency of immunization monitoring systems. Bulletin of the World Health Organization 83, 503-510. 
346 Tao, W., Petzold, M., Forsberg, B.C., 2013. Routine vaccination coverage in low- and

347 middle-income countries: further arguments for accelerating support to child vaccination

348 services. Global health action 6, 20343.

349 United Nations, 2013. The Millenium Development Goals Report, UN, ed, New York.

350 UNPD, 2013. Human Development Report 2013, New York.

351 Van Malderen, C., Ogali, I., Khasakhala, A., Muchiri, S.N., Sparks, C., Van Oyen, H.,

352 Speybroeck, N., 2013. Decomposing Kenyan socio-economic inequalities in skilled birth

353 attendance and measles immunization. International journal for equity in health $12,3$.

354 WHO, UNICEF, World_Bank, 2009. State of the world's vaccines and immunization.

355 Geneva: World Health Organization, 3rd ed.

356 World Health Organization, UNICEF, 2013. Immunization Summary. WHO, Geneva. 
359

360

361

362

363

364

365

\section{FIGURE TITLE}

Figure 1. Study profile

\section{SUPPLEMENTARY MATERIAL}

Supplementary Figure 1. Study area location in Manhiça district, Southern Mozambique 
1096 children under-five residing

in Taninga in September 2012

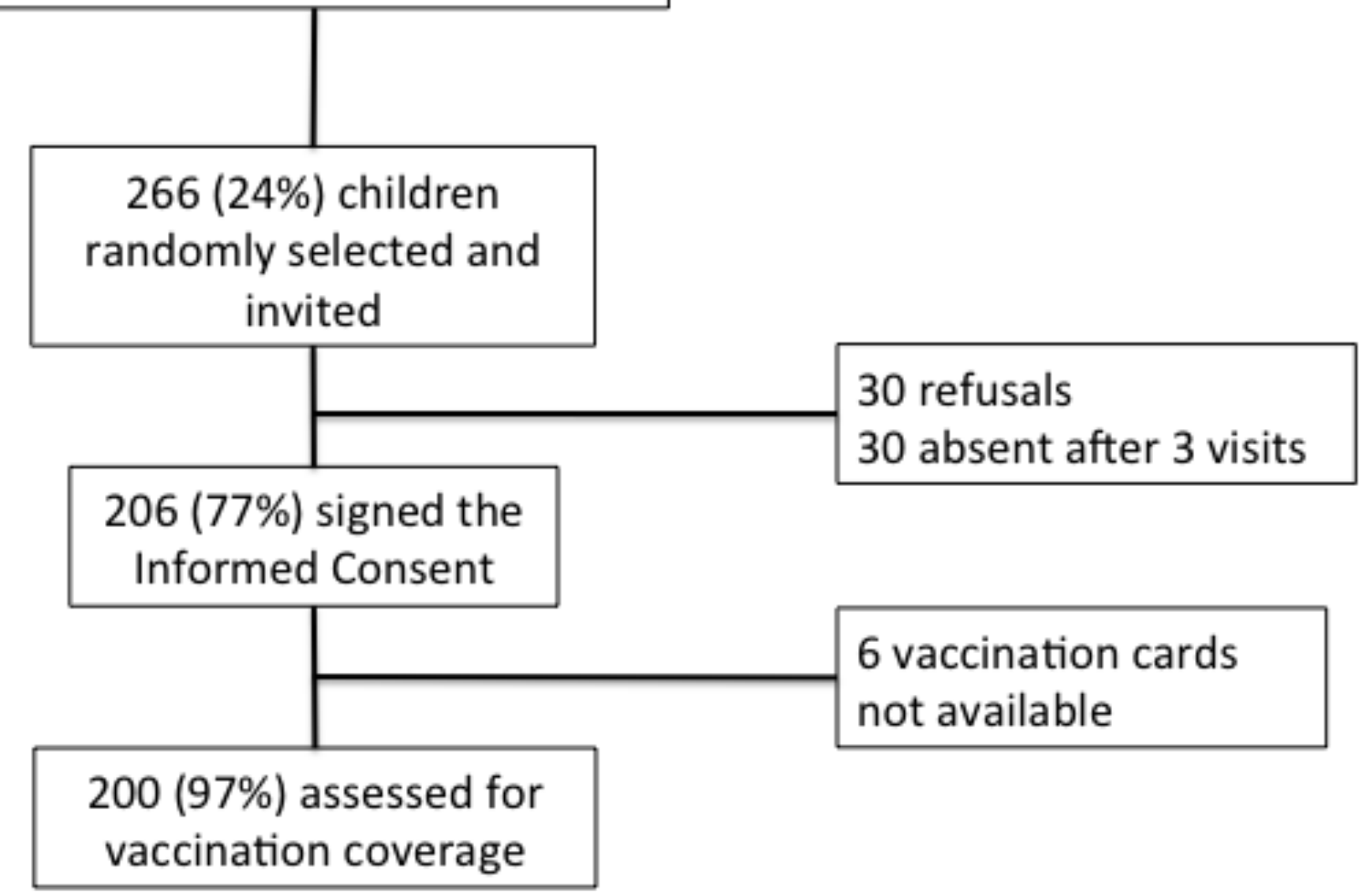

30 absent after 3 visits

vaccination cards

not available 
Table 1. Immunization schedule in Mozambique

\begin{tabular}{lll}
\hline Age & Visit & Antigen \\
\hline Birth & 1 & $\begin{array}{l}\text { BCG, } \\
\text { OPV0 }\end{array}$ \\
6 weeks & 2 & $\begin{array}{l}\text { DTP-HepB1-Hib1*, } \\
\text { OPV1 }\end{array}$ \\
10 weeks & 3 & $\begin{array}{l}\text { DTP-HepB2-Hib2*, } \\
\text { OPV2 } \\
\text { 14 weeks }\end{array}$ \\
9 months & 4 & $\begin{array}{l}\text { DTP-HepB3-Hib3*, } \\
\text { OPV3 } \\
\text { Measles }\end{array}$ \\
\hline
\end{tabular}

$\mathrm{BCG}=$ Bacille Calmette-Guerin; OPV = oral polio vaccine, DTP = Diphteria, Tetanus, Pertussis; HepB = Hepatitis B; Hib = Haemophilus influenza type b

* Hib introduced in August 2009 
Table 2. Descriptive of participants $(\mathrm{N}=206)$

\begin{tabular}{|c|c|}
\hline Variable & Frequency; n(\%) \\
\hline Sex, Male & $108(52.4)$ \\
\hline $\begin{array}{l}\text { Nutritional status (weight-per-height, } \mathbf{Z} \text {-s } \\
\text { Adequate } \\
\text { Mild malnutrition }(-1<\mathrm{Z} \text {-score } \leq-3) \\
\text { Severe malnutrition }(\mathrm{Z} \text {-score }<-3)\end{array}$ & $\begin{array}{l}133(64.9) \\
65(31.7) \\
7(3.4)\end{array}$ \\
\hline $\begin{array}{l}\text { Maternal HIV status } \\
\text { Negative } \\
\text { Positive } \\
\text { Unknown }\end{array}$ & $\begin{array}{l}121(58.7) \\
42(20.4) \\
43(20.9)\end{array}$ \\
\hline Mother's unemployement & $178(86.4)$ \\
\hline Father's unemployement & $25(12.1)$ \\
\hline & Median (interquartile range) \\
\hline Age (months) & $15.5(7.9-23.9)$ \\
\hline Number of children per mother & $3(2-4)$ \\
\hline People living in the household & $7.7(5-9.5)$ \\
\hline Distance to health post (meters) & $1916(1122-2969)$ \\
\hline
\end{tabular}


Table 3. Vaccination coverage for vaccines included in the Expanded Programme on Immunization in children under-five years of age from Taninga $(\mathrm{N}=200)$

\begin{tabular}{ll}
\hline Antigen & Vaccination coverage (95\% CI) \\
\hline BCG & $100 \%$ \\
DTP/HepB +/- Hib & $98 \%(97-99)$ \\
Oral polio & $97.5 \%(96.4-98.6)$ \\
Measles & $96.5 \%(95.2-97.8)$ \\
\hline
\end{tabular}

$\mathrm{BCG}=$ Bacille Calmette-Guerin; DTP = Diphteria, Tetanus, Pertussis; HepB = Hepatitis B; Hib = Haemophilus influenza type b 
Table 4. Socio-economic charateristics, by vaccination status

\begin{tabular}{|c|c|c|c|}
\hline & $\begin{array}{l}\text { Correctly } \\
\text { vaccinated } \\
190(95 \%) \\
\end{array}$ & $\begin{array}{l}\text { Not correctly } \\
\text { vaccinated } \\
10(5 \%)\end{array}$ & p-value \\
\hline \multicolumn{4}{|l|}{ Material of the house; $n(\%)$} \\
\hline Brick & $74(39)$ & $3(30)$ & 0.91 \\
\hline Sheet iron & $113(59.5)$ & $7(70)$ & \\
\hline Shack & $3(1.5)$ & $0(0)$ & \\
\hline \multicolumn{4}{|l|}{ Toilet type; n (\%) } \\
\hline Traditional latrine & $184(96.8)$ & $9(90)$ & 0.17 \\
\hline Improved latrine & $3(1.6)$ & $0(0)$ & \\
\hline None & $3(1.6)$ & $1(10)$ & \\
\hline Kitchen located outside; n (\%) & $187(98.4)$ & $10(100)$ & 0.83 \\
\hline $\begin{array}{l}\text { Average occupation per sleeping room; } \\
\text { median }\left(\mathrm{IQR}^{1}\right)\end{array}$ & $3.5(2.6-4.5)$ & $4(3.3-4.5)$ & 0.93 \\
\hline \multicolumn{4}{|l|}{ Water source; n (\%) } \\
\hline Piped water & $120(63.2)$ & $3(30)$ & 0.036 \\
\hline Well & $70(36.8)$ & $7(70)$ & \\
\hline Electricity unavailable; $\mathbf{n}(\%)$ & $135(71)$ & $9(90)$ & 0.19 \\
\hline \multicolumn{4}{|l|}{ Fuel used to cook; $n(\%)$} \\
\hline Wood & $176(92.6)$ & $10(100)$ & 0.67 \\
\hline Coal & $3(6.8)$ & $0(0)$ & \\
\hline Electricity & $1(0.6)$ & $0(0)$ & \\
\hline $\begin{array}{l}\text { Distance to health post (meters); } \\
\text { median (IQR) }\end{array}$ & 1849 (1095-2934) & $2268(1382-3218)$ & 0.48 \\
\hline \multicolumn{4}{|l|}{ Mother's education; n (\%) } \\
\hline None & $72(37.9)$ & $5(50)$ & 0.47 \\
\hline Primary & $96(50.5)$ & $5(50)$ & \\
\hline Secondary & $22(11.6)$ & $0(0)$ & \\
\hline \multicolumn{4}{|l|}{ Father's education; n (\%) } \\
\hline None & $47(24.7)$ & $3(30)$ & 0.53 \\
\hline Primary & $108(56.8)$ & $7(70)$ & \\
\hline Secondary & $33(17.4)$ & $0(0)$ & \\
\hline Superior & $2(1.1)$ & $0(0)$ & \\
\hline Mother's unemployed; n (\%) & $160(86)$ & $10(100)$ & 0.2 \\
\hline Father's unemployed; n (\%) & $24(13)$ & $1(12.5)$ & 0.96 \\
\hline
\end{tabular}




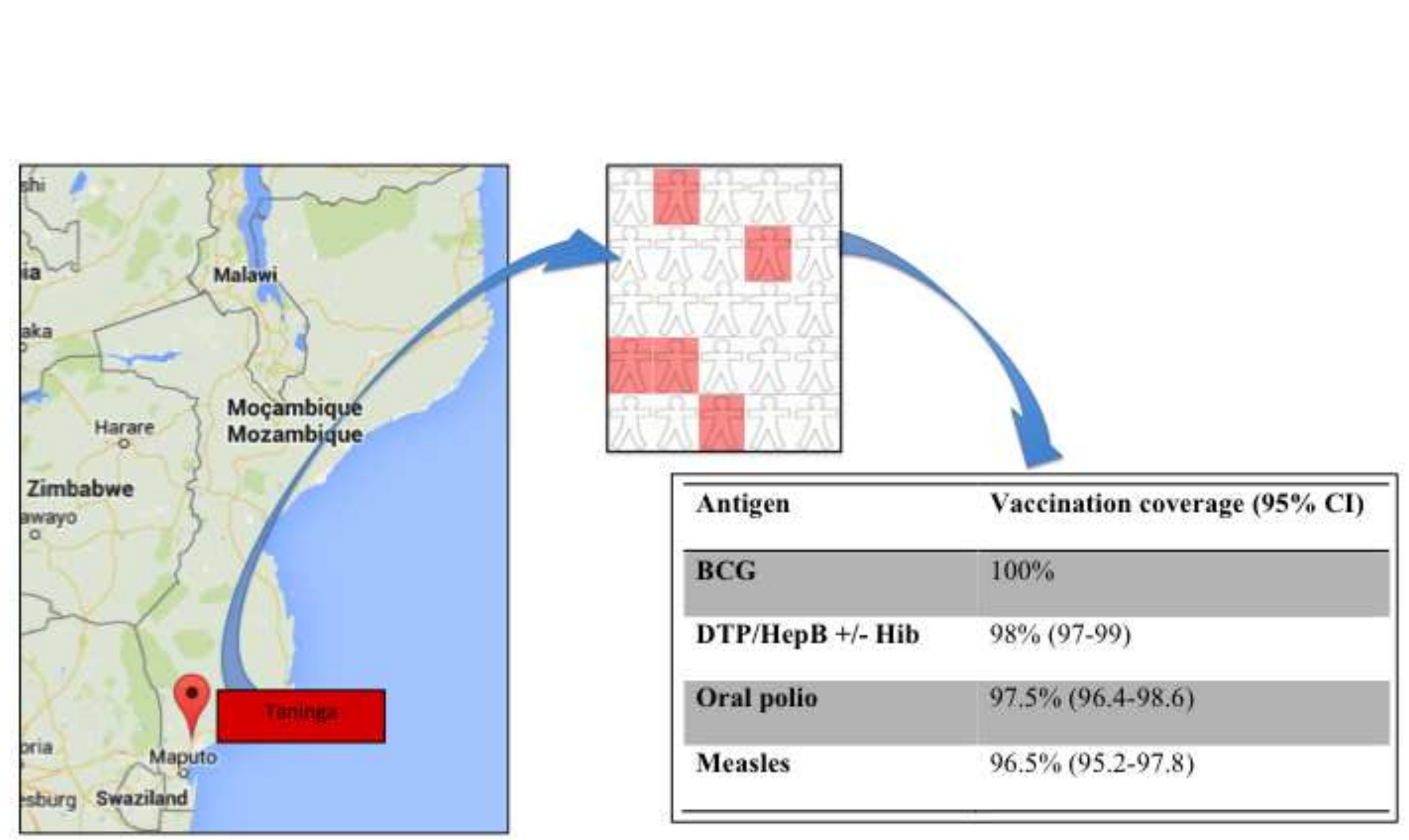

